\title{
Application of a Web-based Monitoring and Control system in Plastic Rotational Moulding Machine
}

\author{
Mário Rodrigues ${ }^{1}$, José Mendes ${ }^{2}$, and Jaime Fonseca ${ }^{3}$ \\ University of Minho \\ 1,2,3 Dept. of Industrial Electronics \\ Campus de Azurem, Portugal \\ Phone: +351253510190 , Fax:+351253510189 \\ e-mail: mario.fernando@iol.pt and \{jaime|jmendes \}@dei.uminho.pt \\ ${ }^{3}$ Addressee for correspondence
}

\begin{abstract}
This paper presents a web based monitoring and control system. The system was applied to an industrial Rotational Moulding Plastic Oven. The developed system provides remote monitoring of several temperature sensors included in the oven, as well as control of the operation cycle in which the oven works. The oven is a rotational machine therefore no cables were able to be used, so it was necessary to use wireless technologies like radio frequency to achieve communication between the machine and the monitoring station. The system architecture and the developed software are presented in this paper.
\end{abstract}

Key Words - Monitoring, Web, Radio Frequency, Teleoperation.

\section{Introduction}

The electronics development as well as the information technologies, especially the internet, brought many news technologies, little wonders of today, unthinkable yesterday and some probably obsolete in a near future.

However, all this technology development only has meaning if applied in the real world. Today, there is a need for automated systems that reduce labour costs, increasing production and providing higher quality results.

In order to achieve those three goals some types of systems have suffered a huge evolution, like systems for remote monitoring and control, teleoperation, vision systems.

Teleoperation systems are everywhere; these kind of systems are applied in many different industry fields, especially in industrial robotics where robots are remotely controlled by users that visualize the environment in which robots operate by a dedicated vision system [1]. This kind of systems can also be integrated with web services which provide real time monitoring and control of a machine process from any place anytime [2] [3].

The rotational moulding, also known as rotational foundry is a polymeric materials transformation process, used for the production of hollow pieces or open pieces, such as tanks, toys, play-grounds, bottles, etc.

The rotational moulding process is composed by four stages as seen in Figure 1:

\footnotetext{
- $\quad$ Charging Mould

- Heating \& Fusion

- Cooling
}

- Unloading/Demoulding

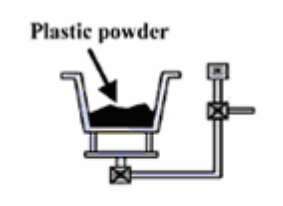

(a) Charging

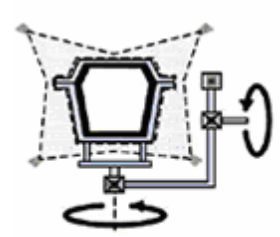

(c) Cooling

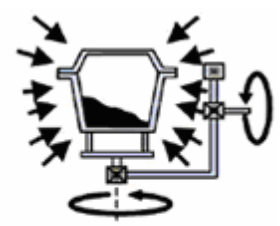

(b) Heating

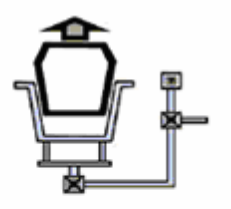

(d) Demolding
Fig. 1: Rotational Moulding Process

This process first appeared in 1947 and suffered a huge evolution since then. In our days the machines that perform this kind of process are highly automated and some of them perform the full process without human intervention. Despites all the automation, the vast majority of these machines requires constant presence of an operator that starts/ends the process and monitores of the process. The human presence, despites being a passive way, is needed.

Plastic rotational moulding is a slow process with many quality specifications, requiring full time attention from worker that supervises/control the process. If one of these machines had a remote monitoring system, the human resources could be better used; a worker could supervise several machines at the same time, because physical presence would no longer be necessary in most of the cases.

The fast growing field of information technologies, especially Internet will definitely help changing this fact. Some machines are now starting to have Ethernet connections, to give the possibility of remote assistance as well as remote programming, control and monitoring.

It is not difficult to forecast that in a not far future all these machines will be fully controlled in a remote way. Humans and machines will interact in different environments like if they were standing in the same place.

This work describes the integration of remote monitoring over the web in a Rotational Moulding Oven. The implemented system, described in this paper, allowed an operator to monitore remotely the temperature in some 
critical spots of the machine and to visualize the environment around the machine.

\section{System Architecture}

The system architecture is shown in figure 2. The system is composed by an Industrial Rotational Moulding Oven (figure 3) equipped with a temperature acquisition system, based on a PLC and radio frequency transceivers, and a monitoring workstation, composed by a $\mathrm{PC}$ with the monitoring software.

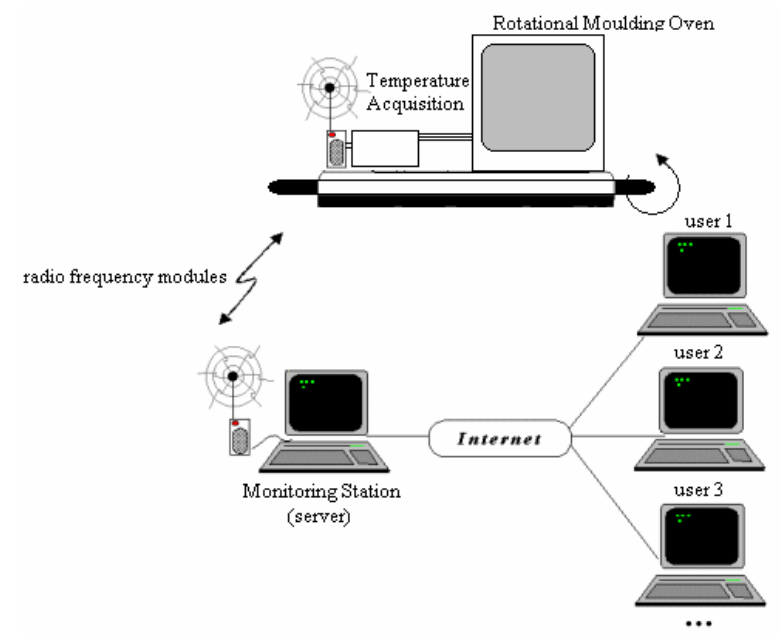

Fig. 2: System architecture.

The simplified layout of the system presented on the figure 2, shows that there is not a physical conection between the machine and the monitoring station. The oven is in permanent movement, translational and rotational, the acquisition equipment is placed inside the machine structure, and wich means that the communications between the machine and the monitoring station cannot use cables. There for, wireless technologies, in this case radio-frequency modules, were used to achieve communications between machine and station.

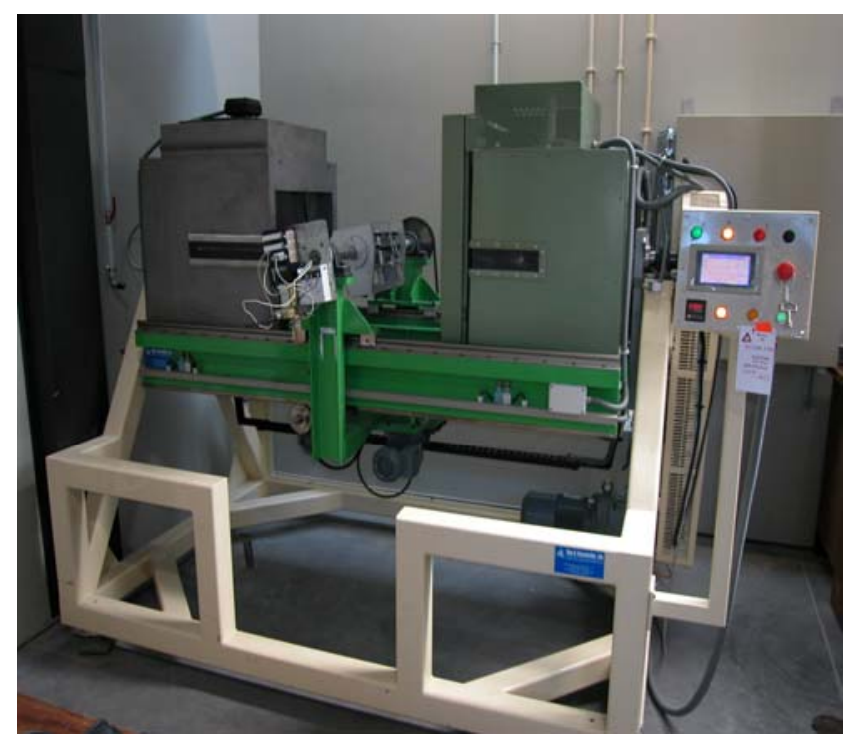

Fig. 3: Rotational moulding oven

\subsection{Acquisition hardware (PLC-CJ1M)}

The physical entity to be measured and displayed in the current case is the temperature provided by several thermocouples placed in several critical points inside the oven. In order to acquire that information it was used a Programmable Logic Controller (PLC) CJ1M from Omron with two Temperature Control Units, the CJ1W-TC001 and $\mathrm{TC} 003$.

This PLC is responsible for the thermocouples measurements, but also for the communications with the monitoring workstation. The program cycle of the PLC starts with reading the information provided by each thermocouple connected to the system and saving that information in a known data memory area. The data collected from the temperature sensors is grouped in a frame that is sent over a serial interface. The serial communications between the acquisition equipment and the Monitoring PC were implemented using wireless technologies, in this case a radio link.

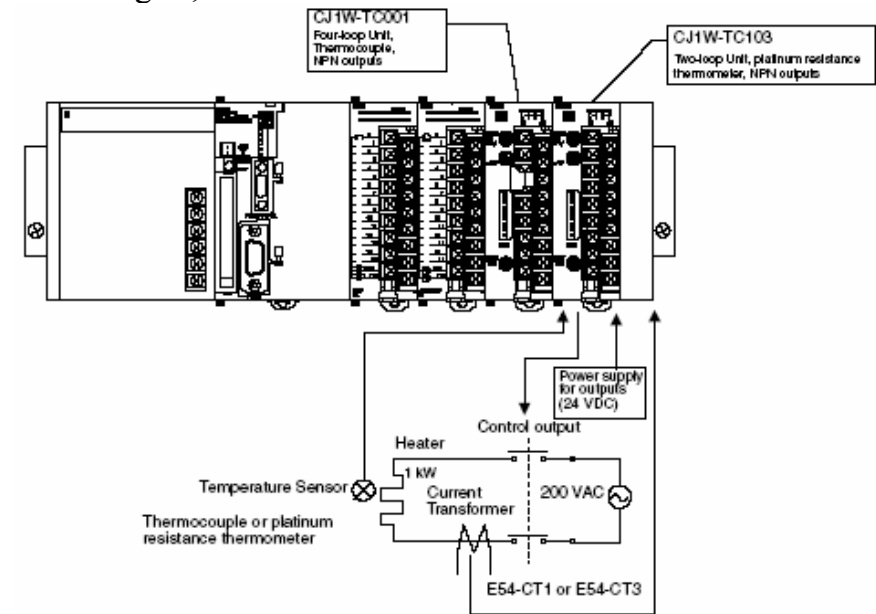

Fig. 4: Temperature acquisition equipment (PLC CJ1M+TC001+TC003)

\subsection{Communication hardware (BIM 433)}

No wires were able to be used because the thermocouples were placed inside the oven that is supported by a rolling structure together with the PLC. For this purpose, two BIM-433 low power UHF data transceiver modules were used to send the data to the monitoring station.

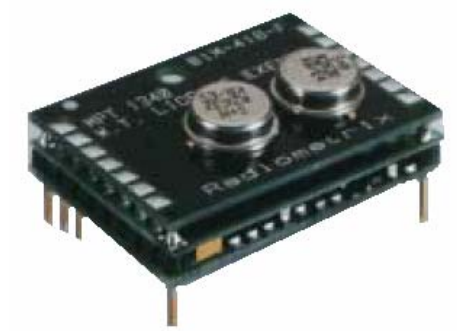

Fig. 5: BIM 433 Radio frequency module.

The BIM 433 is a miniature UHF radio module capable of half duplex data transmission at speeds up to $40 \mathrm{Kbit} / \mathrm{s}$ over distances of 30 metres "indoor" and 120 metres on open ground. 
These modules provide serial communication over a radio frequency link. This means that it is like a virtual serial cable connected between the PC and the temperature control units installed on the oven. Communication can be bi-directional, since each one of the used modules possess both the TXD and RX pins, meaning that they can work either as an emitter or a receiver.

Radio frequency modules are a real advantage in this kind of system, however cable communication has fewer problems with the electromagnetic interferences, which may cause communication errors and reduce reliability of results. It is impossible to physically detect communication errors in these modules. This module only has two communication pins (TXD and RX), so the only possibility to eliminate errors caused by interferences is to implement an error detection algorithm. Such a communication protocol was implemented, in wich the results were either validated or just ignored.

\subsection{Communication protocol}

The radio-frequency modules were used to link the machine to the monitoring station, on top of it. So that it was necessary to implement a communication protocol for further reliability of the serial interface as it operates in a dirty industrial environment.

It was necessary to choose the serial parameters appropriately, specially the communication speed. The system operates mainly in an industrial environment with all sorts of electrical machines around. Due to this fact the baud-rate could not be very high because using higher baud-rate will inevitably cause more transmission errors.

The data sent from the PLC to the monitor station comprises the thermocouples readings. The information of each one is grouped in a frame that will be decoded by the monitor software in the host PC.

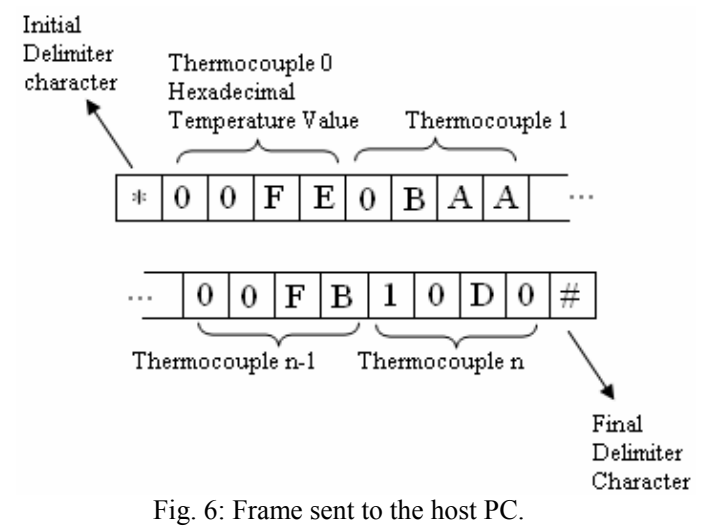

In Figure 6 there is an example of a frame sent by the PLC to the host PC. The frame is composed by two delimiter characters (* and \#), followed by a 16 bit CRC code, whose only function is to detect communication errors that may occur. The temperature information from the thermocouples, four hexadecimal characters per sensor, is between those delimiters characters. The size of the frame is directly proportional to the number of sensors that the system has and it is given by the following expression:

$$
\text { size }(\text { bytes })=4 \times \text { number_sensors }+2
$$

\subsection{Software tool}

The Labview 6.1 toolset was used as software tool to develop the main application in the PC. This software tool is a graphic high level programming language which allows programmers to develop software in a very interactive and graphical way. There are multiple tools, like charts, displays, tabs and others, that allow programmers to create rich graphical user interfaces with little effort, allowing more time for the programmer to concentrate on the core of the application.

In this kind of systems it is important that the application software is not limited to local monitoring and control. It is necessary that users have the possibility of remotely control and monitor the status of the full system. Therefore, two software applications were created, one that runs by itself on the monitoring station and other that runs over a web browser and allows users to visualize in real time mode the temperature as well as real time viewing of the oven and its environment, using for that effect one or more video cameras.

\section{Experimental Results}

The monitoring application allows visualizing in real time mode the temperature information provided by the thermocouples and based on that information the user can control the operation cycle of the oven.

This application not only displays the temperature in several spots of the oven, but also saves the results in a worksheet so that the referred results can be interpreted later using any data analysis software package commercially available.

This application runs in a monitoring station located near the machine (Figure 7). Further, the monitoring PC will also work as a web server, where the second application is placed, allowing users to remotely access all relevant information. This PC is the host, so every operation relative to the system depends on it directly or indirectly.

The web application also integrates control system with vision system, in this way it is possible to control and operate the machine safely and remotely (Figure 8). The user can view the information as well as the images from the oven, this feature certainly allows more flexibility and security in handling of the system.

This application comprises an HTML document that contains the main application panel included in HTML code as an Active $\mathrm{X}$ control.

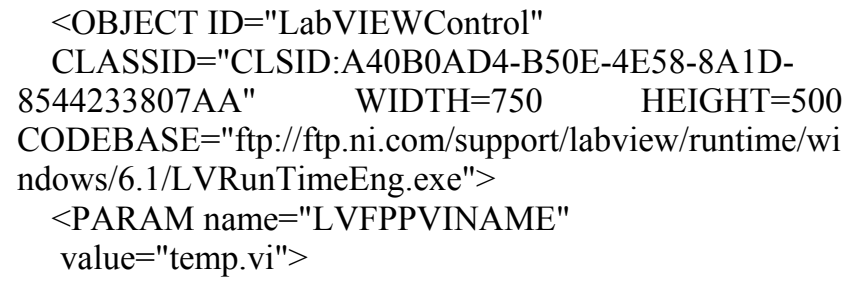


$<$ EMBED SRC $=$ ".LV_FrontPanelProtocol.rpvi"

LVFPPVINAME $=$ "temp.vi" $\quad$ TYPE $=$ "application/ $\mathrm{x}-$ labviewrpvi" $\quad$ WIDTH=750 HEIGHT=500 PLUGINSPAGE="http://digital.ni.com/express.nsf/expres s?openagent\&code $=$ ex $87 \mathrm{~nm} \& "></$ EMBED $>$

$</$ OBJECT $>$

The HTML code can be written by the programmer as a normal web page with the code that is presented above or it can be automatically generated trough the Labview web publishing tool. This tool provides the user that do not have HTML knowledge to build a web page with the program that has been previous developed for local operation.

In this case, it was created a new HTML document so that video monitoring could also be included as an ActiveX object embedded in HTML code.

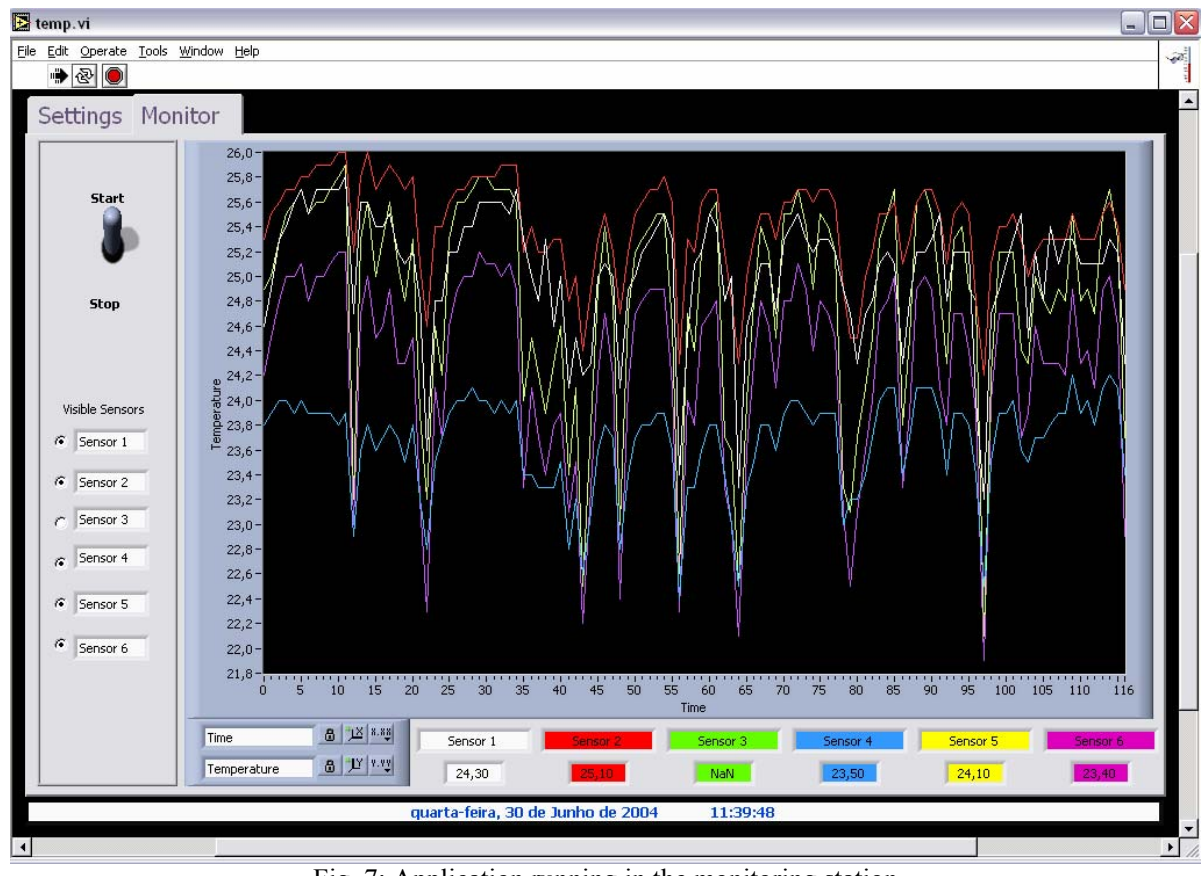

Fig. 7: Application running in the monitoring station

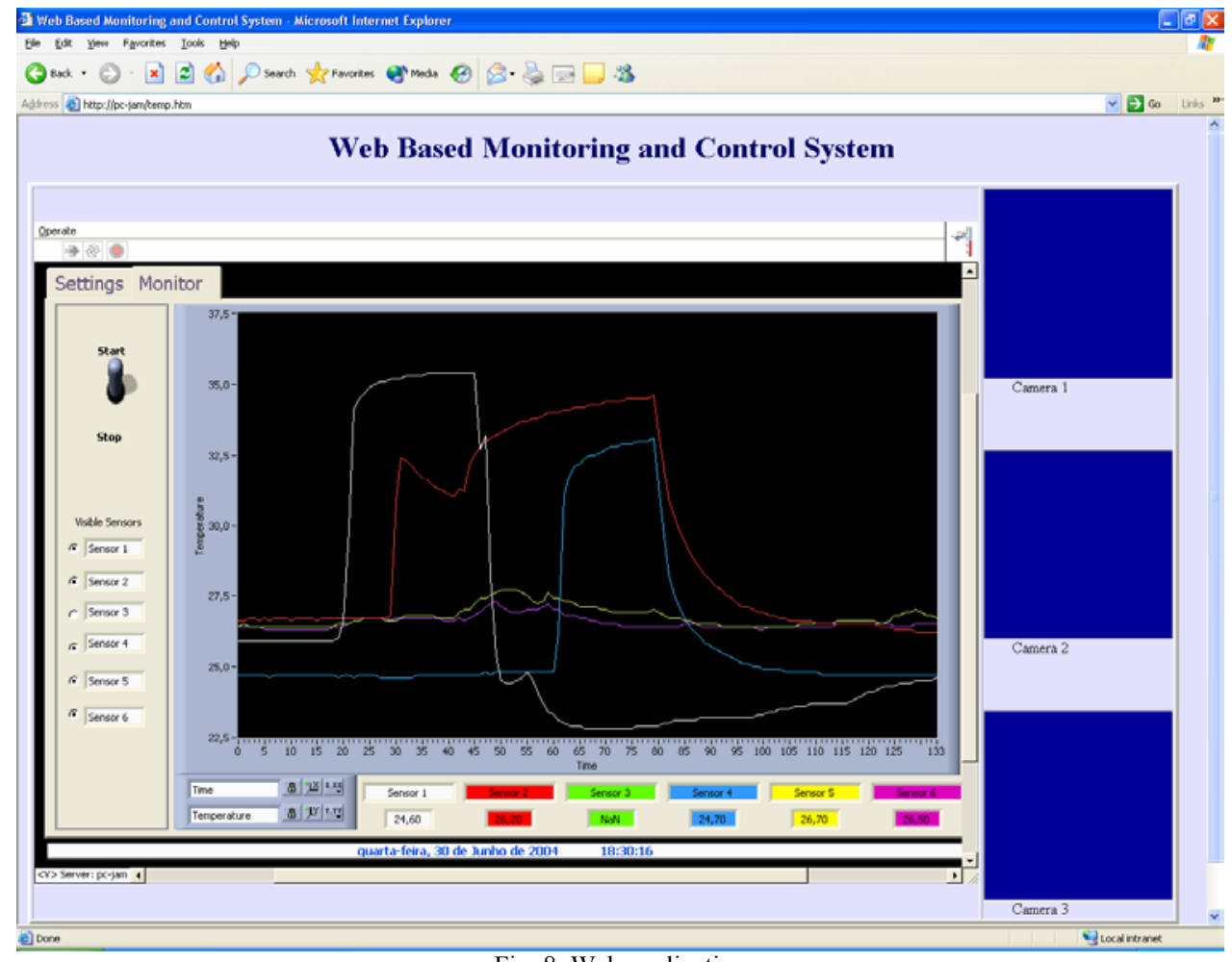

Fig. 8: Web application 


\section{Conclusions}

In this paper, it is presented a Web based Monitoring and Control System applied to the industry of rotational moulding plastic. The implemented system provides a low cost solution to remotely control and monitor the critical parameters of the plastic moulding process.

The developed work allowed bigger flexibility and reliability of the measurements. Before the implementation of this project workers had to periodically register by hand the temperature in several spots and, based on that information, take the appropriate decisions. Now all that process is automatic and the worker only has to visualize the process developing and intervene when needed.

The system was applied to a rotational oven, but the solution can be perfectly adapted and adopted by many other processes where temperature becomes a critical operational factor. Also, this solution was developed in a very short period of time.

\section{Future work}

New monitoring devices will be included in the system; the idea is to provide workers even more mobility. The objective is to use a Personal Digital Assistant -PDA- with the capacities of the described system. Recent PDAs are already equipped with wireless modules like bluetooth that allow them direct access to the internet, directly (internal modem) or over a bluetooth access point.

The next step is to create a software application that runs in a handheld device, the application will access the internet and get the containers of a CGI script that stores all the relevant data to be displayed.

This new feature will provide worker an independence from the $\mathrm{PC}$ and it will allow mobility within the environment in which the machine is placed.

\section{References}

[1] M. Gauß, A. Bürkle, T.Längle, H. Wörn, "An Architecture and Communication Protocol for Interaction of Industrial Robots and Vision Systems", in Proceedings of ICAR 2003 the 11th International Conference on Advanced Robotics Coimbra, Portugal, June 30- July 3, 2003, pp. 625-630.

[2] Ren C. Luo, Fellow, IEEE, Jyh Hwa Tzou, and Yi C. Chang, "Desktop Rapid Prototyping System With Supervisory Control and Monitoring Through Internet", in IEEE/ASME Transactions on Mechatronics, Vol. 6, NO.4, December 2001, pp. 339-409.

[3] Maxwell E. Monteiro; Elton S. Moura, Adrian B. Drago, Luis O. Bonino, Jose G. Pereira, Paulo F. Amaral, Domingos S. Simonetti, Pedro F. Rosa, Geraldo D. Bazelatto, "An InternetBased Power Quality Monitoring System".

[4] National Instruments - Labview, software that powers virtual instrumentation, http://www.ni.com/labview/.

[5] Macromedia - Dreamweaver MX, http://www.macromedia.com/software/dreamweaver. 